\title{
A CMR study assessing aortic valve hemodynamics post-transcatheter aortic valve implantation compared to surgical aortic valve replacement
}

\author{
Timothy Fairbairn ${ }^{1 *}$, Christopher D Steadman², Adam N Mather ${ }^{1}$, Manish Motwani ${ }^{1}$, Daniel Blackman ${ }^{3}$, \\ Sven Plein ${ }^{1,3}$, Gerry P McCann², John P Greenwood ${ }^{1,3}$
}

From 15th Annual SCMR Scientific Sessions

Orlando, FL, USA. 2-5 February 2012

\section{Summary}

Transcatheter aortic valve implantation (TAVI) removes the valvular impedance associated with aortic stenosis but can be complicated by significant aortic regurgitation. Using cardiovascular magnetic resonance we assessed the change in trans-aortic pressure gradient and regurgitant fraction 6 months post-TAVI and compared this to a surgical aortic valve replacement (SAVR) cohort. TAVI was superior at reducing aortic pressure gradient and equal to SAVR at reducing aortic regurgitation at 6 months.

\section{Background}

Transcatheter aortic valve implantation (TAVI) is a rapidly developing procedure for the treatment of patients with severe aortic stenosis (AS) at high surgical risk. A reduction in trans-aortic pressure gradient is important to allow the ventricle to reverse remodel, but post-operative aortic regurgitation is a commonly reported complication. The quantification of aortic regurgitation (AR) by echocardiography and fluoroscopic techniques is limited, in part due to the paravalvular nature of regurgitation. We aimed to use cardiovascular magnetic resonance (CMR) imaging to determine the change in trans-aortic pressure gradient and accurately quantify paravalvular and valvular regurgitation postTAVI compared to surgical aortic valve replacement (SAVR).

${ }^{1}$ Multidisciplinary cardiovascular research centre, University of Leeds, Manchester, UK

Full list of author information is available at the end of the article

\section{Methods}

Fifty high-risk patients (EuroSCORE $\geq 20$ ) with severe AS (peak velocity $>4 \mathrm{~m} / \mathrm{s})$ underwent TAVI $(\mathrm{n}=25)$ or SAVR $(\mathrm{n}=25)$. TAVI was performed using the Medtronic CoreValve prosthesis $(26 \mathrm{~mm}(20 \%)$ and $29 \mathrm{~mm}$ (80\%). Bioprosthetic SAVR of varying sizes $(18-26 \mathrm{~mm})$ were used in $24(96 \%)$ and mechanical $1(4 \%)$ patients. Baseline and 6 month post-operative scans were performed on 1.5T systems (Phillips Intera or Siemens Avanto) using identical protocols. Through-plane velocity encoded (VENC) phase contrast imaging was performed perpendicular to the aortic valve jet at the aortic sinotubular junction (Figure 1, VENC $250-500 \mathrm{~cm} / \mathrm{s}$, retrospective gating, slice thickness $6 \mathrm{~mm}, 40$ phases, FOV $340 \mathrm{~mm}$ ). Aortic flow was quantified using QFlow (Medis, Netherlands) to provide a peak forward flow velocity $(\mathrm{m} / \mathrm{s})$, forward flow volume $(\mathrm{ml})$, backward flow volume $(\mathrm{ml})$ and calculation of regurgitant fraction (\%).

\section{Results}

AS severity was similar between the TAVI and SAVR groups ( $50 \pm 16$ vs. $55 \pm 20 \mathrm{mmHg}, \mathrm{P}=0.7$ respectively). Post-operatively the trans-aortic pressure gradient was significantly lower in both groups (TAVI $21 \pm 8 \mathrm{mmHg}$, $\mathrm{P}<0.001$; SAVR $35 \pm 13 \mathrm{mmHg}, \mathrm{P}<0.001$ ), although when compared to SAVR the TAVI group had a significantly greater reduction in their pressure gradient $(\mathrm{P}=0.017)$. Aortic regurgitant fraction at baseline was similar between the TAVI and SAVR groups $(16 \pm 11$ vs. $18 \pm 7 \%$, $\mathrm{P}=0.4$ respectively). Valve replacement/implantation resulted in an $8 \%$ reduction of AR in both groups, reaching statistical significance in the TAVI group 

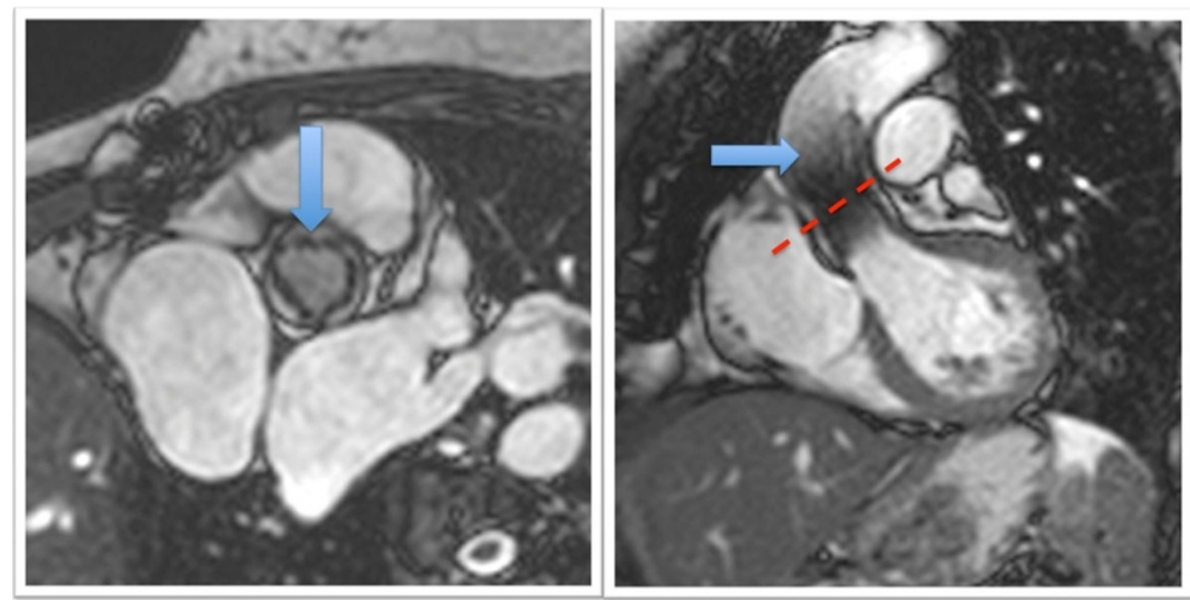

Figure 1 Short axis and coronal views of the CoreValve ${ }^{\mathrm{TM}}$ prosthesis (blue arrow). The typical scanning position for phase contrast imaging is represented by the red dashed line.

$(\mathrm{P}=0.003)$ but not in the SAVR group $(\mathrm{P}=0.09)$. However, comparison of the procedures by 2-way ANOVA showed no difference $(\mathrm{P}=0.46)$ in their efficacy to reduce AR.

\section{Conclusions}

TAVI results in a greater improvement in aortic valve pressure gradient compared to SAVR and reduces aortic regurgitation to a similar degree. This is clinically important as a lower aortic pressure gradient may encourage greater left ventricular (LV) reverse remodeling and post-operative aortic regurgitation may result in pulmonary oedema and impaired ventricular remodeling.

\section{Funding}

None.

\section{Author details}

${ }^{1}$ Multidisciplinary cardiovascular research centre, University of Leeds,

Manchester, UK. ${ }^{2}$ Cardiovascular sciences, University of Leicester, Leicester,

UK. ${ }^{3}$ Cardiology, Leeds General Infirmary, Leeds, UK.

Published: 1 February 2012

doi:10.1186/1532-429X-14-S1-P96

Cite this article as: Fairbairn et al: A CMR study assessing aortic valve hemodynamics post-transcatheter aortic valve implantation compared to surgical aortic valve replacement. Journal of Cardiovascular Magnetic Resonance 2012 14(Suppl 1):P96.

\section{Submit your next manuscript to BioMed Central} and take full advantage of:

- Convenient online submission

- Thorough peer review

- No space constraints or color figure charges

- Immediate publication on acceptance

- Inclusion in PubMed, CAS, Scopus and Google Scholar

- Research which is freely available for redistribution 